\title{
RETRACTED ARTICLE: Discovery of iron-sensing bacterial riboswitches
}

\author{
Siladitya Bandyopadhyay ${ }^{1,2}$, Susmitnarayan Chaudhury ${ }^{1}{ }^{1}$, Dolly Mehta ${ }^{1,2}$ and Arati Ramesh ${ }^{1}{ }^{凶}$
}

As of 29 June 2021, the authors are retracting this Article because of issues with data integrity and reproducibility. Specifically, native gel analyses of natural and engineered Sensei RNAs (Figs. 3e and 5c) raised concerns of gel background similarities from different experiments. On learning this, A.R. requested an independent researcher with expertise in RNA research to attempt to replicate the same experiments. We now find that these data are not reproducible. Further, in the in vivo expression analyses, the RpoA control and $+\mathrm{J} 41$ western blots (part of Fig 4, Extended Data Fig. 8) raise concerns of gel band duplications. In addition, ITC curves (shown in Fig. If and Extended Data Fig. 4b) have raised concerns of inconsistent spacing between successive injections. We no longer have access to the raw data for these experiments and hence are unable to verify the salt-dependent binding affinities of Sensei RNAs.

Given that the key data supporting the iron ion-induced structural changes in Sensei RNAs-a central conclusion of the original paper-are now called into question, we believe that retraction of the work in its entirety is essential. The authors deeply regret this situation and apologize to the scientific community. All authors of the original study have agreed to retract the article and with this retraction text.

A.R. informed the institute about these concerns regarding data integrity, following which an independent inquiry was conducted. The inquiry committee also recommends that the Article be retracted.

The full text of the retracted article is available as Supplementary Information.

Received: 2 December 2019; Accepted: 26 August 2020;

Published online: 5 October 2020

(c) The Author(s), under exclusive licence to Springer Nature America, Inc. 2020 